\title{
Aconselhamento em amamentação e sua prática
}

\author{
The practice of breastfeeding counseling
}

\author{
Lais Graci dos Santos Buenoㄹ, Keiko Miyasaki Teruya²
}

\section{Resumo}

Objetivo: Proporcionar aos profissionais de saúde informações referentes ao aconselhamento em amamentação com base em teoria e prática.

Fontes dos dados: Foram selecionadas, analisadas e utilizadas informações relevantes ao tema provindos dos sistemas MEDLINE, Bireme, Lilacs e sites relevantes da Internet, além de revistas científicas, livros técnicos, dissertações e teses e publicações de organismos nacionais e internacionais. As principais fontes foram: publicações da Organização Mundial da Saúde (1993) e experiências e vivências clínicas na assistência a mãe/filho/família das autoras.

Síntese dos dados: $O$ pediatra treinado em "aconselhamento em amamentação" pode desempenhar papel relevante no aumento das taxas e duração do aleitamento materno. A principal fonte de treinamento é o curso de 40 horas elaborado pela Organização Mundial da Saúde, em 1993, que emprega uma importante estratégia didática de comunicação entre profissionais de saúde e a mãe para a proteção, promoção e apoio ao aleitamento materno. Aconselhamento em amamentação implica o profissional escutar, compreender e oferecer ajuda às mães que estão amamentando, fortalecendo-as para lidar com pressões, promovendo sua autoconfiança e auto-estima e preparandoas para a tomada de decisões.

Conclusão: Evidências científicas comprovam a efetividade do aconselhamento em amamentação. Seu conhecimento e prática pelos profissionais de saúde constitui um importante instrumento para o aumento das taxas e duração da amamentação.

J Pediatr (Rio J). 2004;80(5 Supl):S126-S130: Amamentação, aconselhamento, aconselhamento em amamentação, aleitamento materno, educação médica.

\begin{abstract}
Objective: To provide health professionals with information on theory and practice of breastfeeding counseling.

Sources of data: MEDLINE, Bireme library, Lilacs, relevant Internet websites, scientific journals, technical books, essays, theses, and national and international publications were selected, studied and used to provide information on the topic. The most important sources of data were: a publication by the World Health Organization (WHO - 1993) and the authors' experience and clinic practice in the assistance of mothers, children and families.
\end{abstract}

Summary of the findings: A trained pediatrician plays an important role in the increase of breastfeeding rates and its duration. To improve this performance, in 1993, WHO designed a 40-hour course using an important didactic strategy aimed at health professionals and mothers. The goal was to protect, promote and support maternal nursing. It is a professional way of dealing with the mother by listening and trying to understand her, offering her help on planning, taking decisions, and getting strength on how to deal with pressures, thus increasing her confidence and self-esteem.

Conclusion: Scientific evidences prove the effectiveness of Breastfeeding Counseling. Moreover, health professional's knowledge and practice are very important to increase breastfeeding rates.

J Pediatr (Rio J). 2004;80(5 Supl):S126-S130: Breastfeeding, counseling, breastfeeding counseling, maternal nursing, medical education, pediatrician.

\section{Introdução}

A espécie Homo sapiens - a mais adaptável da escala zoológica - encontrou desde cedo fontes alternativas ao leite humano para alimentar sua cria ${ }^{1}$. Essa prática tem sido intensamente explorada, levando o homem a distanciar-se cada vez mais de sua condição de mamífero ${ }^{2}$. Paga-se um alto preço por essa quebra de paradigma, pois o leite

1. Professora de Pediatria, Curso de Ciências Médicas, Centro Universitário Lusíada (UNILUS), Santos, SP.

2. Doutora em Medicina Preventiva pela USP.

Como citar este artigo: Bueno LGS, Teruya KM. Aconselhamento em amamentação e sua prática. J Pediatr (Rio J). 2004;80(5 Supl):S126S130.

materno, além de diminuir o risco de infecção e até mesmo de morte infantil, aumenta o vínculo mãe-filho, o que possibilita uma melhor qualidade de vida futura, entre outras vantagens ${ }^{3-15}$. O aprimoramento das fontes alternativas ao leite materno explica o declínio da duração e das taxas de amamentação no mundo, sobretudo no século passado ${ }^{16}$. Nas capitais brasileiras, a mediana de amamentação é de 10 meses. No entanto, a mediana de amamentação exclusiva é de apenas 23 dias, havendo variações regionais ${ }^{17}$.

Para reverter essa situação, várias ações têm sido propostas e implementadas por grupos internacionais, como a Organização Mundial da Saúde (OMS), o Fundo das Nações 
Unidas para a Infância (UNICEF), a Academia Americana de Pediatria e o Colégio Americano de Obstetrícia e Ginecologia18-20, e nacionais, tais como o Ministério da Saúde, o Instituto da Saúde de São Paulo, secretarias de estado21, a Sociedade Brasileira de Pediatria, dentre outras. Essas ações incluem educação em amamentação, treinamento de profissionais de saúde e aconselhamento em amamentação, entre outras.

Em relação à educação em amamentação, uma pesquisa da Organização Pan-Americana da Saúde (OPAS) e da OMS realizada no Brasil em 1994 constatou que os cursos de medicina, que contam com cerca de 8.345 horas em média, dedicam apenas 26 horas ( $0,13 \%$ da carga horária total) ao ensino do aleitamento materno. Desde então, tem havido progressos quanto à carga horária e ao ensino do manejo do aleitamento materno, graças aos esforços do Ministério da Saúde com seus centros de referência em treinamento de aleitamento e também de alguns dos órgãos citados.

O pediatra treinado em aleitamento materno desempenha um importante papel na promoção da amamentação, influenciando diretamente sua taxa e duração 22-27. Em sua formação tradicional, o pediatra é treinado para detectar problemas e resolvê-los; usa, para o seu raciocínio clínico, a queixa verbalizada pela mãe, porém nem sempre atinge o problema real vivenciado por ela. A mãe, ao procurá-lo, traz a expectativa de uma boa assistência e da resolução de seus problemas, mas freqüentemente não encontra espaço para expor seus sentimentos e contextualizar suas dificuldades, talvez pela falta de domínio do profissional de como fazer a ponte da teoria à prática. Sendo assim, o momento entre o pediatra e a mãe (que deveria ser vivido na sua plenitude) não se efetiva satisfatoriamente. Dentro desse cenário, com freqüência se observam as seguintes situações: mães que começam a amamentar de maneira satisfatória, mas iniciam a alimentação complementar e/ou param de amamentar poucas semanas após o parto; e pediatras, mesmo conhecedores do manejo da amamentação, que nem sempre conseguem dar ajuda satisfatória.

Saber compreender mais profundamente os problemas que cercam uma consulta de maneira a diagnosticá-los corretamente e, assim, ajudar efetivamente uma mãe a resolver seu problema é o cerne do aconselhamento ${ }^{28}$. O aconselhamento em amamentação é uma ferramenta especialmente recomendada desde 1994 pela Força-Tarefa Canadense no Cuidado da Saúde Preventiva (CTFPHC) e pela Força-Tarefa Americana de Serviços Preventivos dos Estados Unidos (USPSTF). As recomendações são baseadas em evidências de revisões da efetividade das intervenções de promoção do aleitamento materno no mundo. As evidências mostram que as taxas e o tempo de duração da amamentação aumentam quando a mulher recebe aconselhamento em amamentação 29-31.

\section{Aconselhamento}

Aconselhamento é definido por McKinney et al. ${ }^{32}$ como "uma relação interpessoal na qual o conselheiro assiste o indivíduo na sua totalidade psíquica a se ajustar mais efetivamente a si próprio e ao seu ambiente". É considera- do, ainda, como "ajuda na tomada de decisões das pessoas para resolverem os seus próprios problemas, abrangendo informações objetivas que possibilitam uma melhor utilização dos recursos pessoais" 33 .

É importante entender a diferença entre o simples ato de aconselhar e aconselhamento. Aconselhar ou dar conselho é dizer à pessoa o que ela deve fazer; aconselhamento é uma forma de atuação do profissional com a mãe onde ele a escuta, procura compreendê-la e, com seus conhecimentos, oferece ajuda para propiciar que a mãe planeje, tome decisões e se fortaleça para lidar com pressões, aumentando sua autoconfiança e auto-estima 28 .

\section{Aconselhamento em amamentação no Brasil}

Desde 1995 vem sendo implementado o curso de "Aconselhamento em Amamentação: um Curso de Treinamento", com o suporte do Ministério da Saúde, de secretarias de saúde e do Instituto de Saúde de São Paulo. O curso nasceu da iniciativa do Programa de Controle das Doenças Diarréicas (Control of Diarrhoeal Diseases - CDD) em colaboração com UNICEF/OMS, os quais idealizaram e implantaram o curso, testado pela primeira vez em 1991 nas Filipinas, em 1992 na Jamaica e em 1993 em Bangladesh. Desde então, vários países no mundo adotaram esse curso, que tem como objetivo treinar o profissional de saúde em algumas habilidades específicas para facilitar a comunicação e atingir uma ação construtiva, considerando as bases fisiológicas da lactação. O curso conta com uma carga horária de 40 horas e oferece estratégia didática de comunicação entre profissionais de saúde (conselheiros) e a mãe 28 .

Uma avaliação sobre o curso de aconselhamento em amamentação feita no Brasil concluiu que os participantes adquirem habilidades de aconselhamento; porém, para que os mesmos passem a aplicar o aprendizado na prática, há necessidade de reforçar o manejo clínico da lactação e também de uma supervisão continuada ${ }^{34}$.

As habilidades recomendadas no aconselhamento em amamentação da OMS/UNICEF são resumidas na Tabela 1.

\section{Aplicações práticas do aconselhamento em amamentação \\ Pré-natal com aconselhamento em amamentação}

Durante a gestação, a mulher encontra-se numa situação diferente da habitual, com suas dúvidas, insegurança e medo. Isso a torna mais sensível e suscetível frente às pressões de familiares, profissionais de saúde e amigos quanto à sua capacidade de amamentar. Além disso, a mãe pode estar em conflito consigo mesma sobre a decisão de amamentar. Nesse contexto, a mãe pode facilmente perder sua confiança e auto-estima e estar muito propensa a oferecer mamadeira ao seu bebê.

A mãe com auto-estima assegurada é capaz de resistir a pressões contra a amamentação. As seguintes recomendações são particularmente úteis no acompanhamento pré-natal:

- Dar muita atenção aos sentimentos da mãe, respeitando sua opção na escolha do que é melhor para ela e seu filho 
Tabela 1 - Habilidades para o aconselhamento em amamentação

Habilidades de ouvir e aprender

Use comunicação não-verbal útil

Mantenha a cabeça no mesmo nível

Preste atenção

Remova barreiras

Dedique tempo

Toque de forma apropriada

Faça perguntas abertas

Repita o que a mãe diz com suas palavras

Use expressões e gestos que demonstrem interesse

Demonstre empatia - mostre que você entende como a mãe se sente

Evite palavras que demonstrem julgamento

\section{Habilidades para aumentar a confiança e dar apoio}

Aceite o que a mãe pensa e sente

Reconheça e elogie o que a mãe estiver fazendo certo

Dê ajuda prática

Dê poucas informações, selecionando aquelas que são relevantes Use linguagem simples

Dê sugestões, e não ordens

e não induzindo preocupações ou dúvidas sobre sua capacidade de produzir leite.

- Deixar que a decisão final seja tomada pela mãe e mostrar que ela é capaz de optar sobre o que é melhor para ela e seu filho, cabendo ao profissional dar sugestões e informações relevantes, com evidências científicas, numa linguagem simples e clara. A mãe sempre tem o seu saber, cabendo ao profissional compartilhar com ela seus conhecimentos sobre amamentação.

- Reconhecer o real motivo de sua consulta. A identificação da queixa real (que nem sempre é a relatada) é o ponto-chave para sugestões corretas sobre amamentação.

- Corrigir idéias erradas e dar informações corretas de maneira positiva, sem que soem como críticas. Ao dar informação, o pediatra se apresenta como um expert no assunto. Porém, discorrendo excessivamente sobre a questão, suas informações e sugestões podem não ser bem compreendidas nem tampouco acatadas pela mãe. Assim, o importante é selecionar apenas uma ou duas informações relevantes de modo positivo e de modo tal que ela perceba o que deve ser modificado, sempre mantendo uma atitude humilde.

- Fortalecer a compreensão da importância da consulta. Esta pode ter vários significados para a mãe: interromper a sua rotina, expor seus sentimentos, aguardar assistência, despender dinheiro e tempo. Uma simples consulta é sempre muito importante para a mãe que busca apoio e/ou orientação.

- Estar atento ao comportamento verbal e não-verbal tanto da mãe quanto do profissional. Este é o primeiro passo da consulta, que, por vezes, desenvolve-se sem a percepção da diferença contida nas entrelinhas da comunicação do olhar sem ver e do ouvir sem escutar. Mesmo antes de falar ou mesmo sem nada falar, o profissional pode facilitar a comunicação ou, ao contrário, provocar o desinteresse da mãe. Assim, a comunicação entre o profissional e a mãe numa consulta pode ocorrer sem expressão verbal, mas por meio de expressão corporal. Com forte conviç̧ão, de modo natural e por uma representação corporal, a seguinte mensagem deve ser passada para a mãe: "eu estou interessado em recebê-la e em escutar o que você quer contar para mim".

- Manter uma corrente de atenção desde a porta de entrada do hospital/unidade básica até a saída da mãe. Nessa atenção se insere o acolhimento, que é o primeiro passo para o estabelecimento da boa relação médicopaciente.

Uma técnica utilizada no Centro de Lactação de Santos (HGA/UNILUS) no acompanhamento pré-natal é a implementação da dinâmica de grupo ${ }^{33}$ nas salas de espera com as gestantes e acompanhantes. A apresentação de todos os participantes é importante para o aquecimento da reunião. Todos os participantes devem sentir-se inclusos, com liberdade para expor suas dúvidas, aprender com as experiências e vivências dos outros, resolver os problemas e construir sua decisão sobre a amamentação. Para atingir um melhor aproveitamento, o coordenador da dinâmica de grupo deve lembrar e explicar em linguagem simples as informações relevantes não mencionadas durante a reunião, sem exceder 30 minutos de duração.

\section{Sala de parto com aconselhamento em amamentação}

Na sala de parto existe a recomendação de que mãe e filho não devem ser separados após o nascimento, a não ser que exista uma razão médica aceitável ${ }^{35}$. O pediatra, ao assistir o recém-nascido (RN) na sala de parto, poderá estar frente a duas situações quanto ao apoio à amamentação: parturiente preparada durante o acompanhamento prénatal e parturiente não preparada.

Para a parturiente não preparada, recomenda-se uma fala prévia sobre a relevância do contato precoce (na primeira hora pós-parto) pele a pele/olhos nos olhos, sendo sugerido que esse contato ocorra ao nascer. A linguagem deve ser simples e direta. Termos técnicos podem não ser entendidos pelas mães. Em vez de dar ordens, deve-se sempre sugerir. As ordens diminuem a autoconfiança e desviam a tomada da decisão que cabe à mãe.

Caso a parturiente não preparada aceite a sugestão, a assistência daí em diante será idêntica à oferecida para parturientes preparadas. Deve ser oferecido todo apoio e ajuda prática para que se estabeleça o contato precoce. Por exemplo: ajudar a mãe a segurar o RN para que o contato pele a pele ocorra. Uma ajuda prática pode desencadear na mãe, além de sentimento de gratidão, uma abertura de comunicação com o profissional. A mãe, ao se sentir confortável, fica atenta às informações e sugestões do profissional. Por outro lado, quando a mãe está desconfortável, cansada, com sede ou já recebeu muitas informações, uma ajuda prática é melhor que muitas palavras. 
É imprescindível que seja respeitado o ritmo desenvolvido pela mãe e seu filho nesse contato, para assegurar o sucesso do passo seguinte: a mãe colocar o recém-nascido para mamar espontaneamente.

\section{Amamentação no alojamento conjunto com aconselhamento}

Quando o profissional de saúde se apresenta com um sorriso, com um meneio da cabeça, demonstra que está ali para auxiliar, abrindo um canal de comunicação positivo e bastante propício para a promoção da amamentação.

No puerpério imediato e nos primeiros dias pós-parto, a habilidade mais importante do aconselhamento é a ajuda prática. Por exemplo: observar o entorno da mãe para que ela se acomode e descanse, além de sentir-se apoiada (travesseiros, poltronas, cadeiras, oferecer água, providenciar analgésico para dor, etc.).

No alojamento conjunto, outra habilidade importante do aconselhamento é a empatia. Ela é a chave do processo de aconselhamento e, ao mesmo tempo, a chave de todo o trabalho de identificação e compreensão entre pessoas; trabalha sentimentos, e não apenas conversa sobre eles. Esse processo visa estimular a autodescoberta da mãe. A empatia não é um processo mágico, muito embora seja misterioso. Parece ser de difícil compreensão exatamente por ser tão comum e fundamental.

Empatia não deve ser confundida com simpatia (que denota sentir com sentimentalidade); esta, ao invés de facilitar, pode bloquear a comunicação, deslocando o foco de atenção da mãe. Na consulta onde prevalece a simpatia, o pediatra sente pelo que acontece à mãe e olha a situação sob seu ponto de vista. Já na empatia, ele escuta a mãe e demonstra a ela que entendeu seus sentimentos, sob do ponto de vista dela; o foco deve se manter na mãe e em seus sentimentos. O relacionamento mãe/profissional é fortalecido quando a empatia é praticada, a mãe é escutada com atenção e é elogiada no que faz certo.

Outras habilidades do aconselhamento a serem desenvolvidas, principalmente para uma melhor observação e avaliação das mamadas, incluem: sugerir - e não ordenar - que a mãe coloque o RN para mamar; avaliar uma mamada inteira, sem demonstrar pressa; e intervir só quando for solicitado e/ou autorizado pela mãe.

\section{Seguimento da amamentação com aconselhamento}

Para a manutenção da amamentação, a mãe precisa receber apoio e ajuda centrada nas dificuldades específicas ou nas suas crises de autoconfiança. No seguimento, a atenção ao acolhimento é semelhante à do acompanhamento pré-natal. A dinâmica de grupo antes das consultas oferece às mães informações relevantes, deixando-as mais tranqüilas e facilitando a comunicação com o profissional durante a assistência.

A ajuda prática também é importante e se traduz em diferentes fases da assistência, como: ter porteiros treinados para receberem as mães com sorriso e carinho, indicando o local de assistência; evitar burocracia ou fila de espera; acomodar as mães de maneira adequada durante a espera. Outra ajuda prática é estabelecer o hábito de oferecer às mães lanche, suco ou apenas água. Após a dinâmica, segue-se uma consulta individual onde atitudes facilitadoras devem ser utilizadas (Tabela 1 ).

O modo como as perguntas são formuladas é importante. Perguntas abertas, além de estimular a mãe a falar mais e a colocar o que realmente sente, racionalizam o tempo de consulta. As perguntas abertas geralmente iniciam a frase com as seguintes palavras: "como", "que", "quem", "onde", "de que modo", "em que", etc. As perguntas fechadas, cujas respostas se resumem por um "sim" ou "não", além de induzir a uma informação imprecisa, podem bloquear a comunicação. Entretanto, às vezes as perguntas fechadas são necessárias, como, por exemplo, em: "Fez acompanhamento pré-natal?". Perguntas específicas dão continuidade à comunicação; exemplo: "Quando você iniciou a amamentação?". No entanto, às vezes verdadeiras inquisições são feitas com as mães, obtendo-se respostas inúteis e levando-as a falar menos.

O profissional, ao devolver para a mãe o que ela disse, demonstra que entendeu o que ela está dizendo. Sendo assim, ela provavelmente falará mais sobre o assunto e poderá ajudar a direcionar a conversa para fatos que ele precisa conhecer mais. Um simples meneio de cabeça, sorriso ou respostas simples como "Ah é?", "Aha!", "Mmm", "Nossa!", "E aí?", "Sei, sei", etc. são maneiras de demonstrar que o profissional está escutando e que a mãe pode contar com ele, além de estimular a mãe a falar.

Uma mãe que amamenta facilmente perde a confiança em si mesma e pode se tornar suscetível à pressão de familiares e conhecidos para que desmame. É importante que o profissional faça a mãe se sentir confiante e bem consigo mesma, evitando o emprego de certas palavras (certo, errado, bem, mal, bastante, adequado, direitinho, normalmente, suficiente, problema) que podem ter conotação de julgamento.

Todas as habilidades do aconselhamento devem ser atentamente usadas na consulta, com ênfase na empatia: aceitar o que a mãe diz, não julgá-la, não "cobrar" dela posturas e atitudes frente à amamentação, elogiar, informar e sugerir para que a mãe possa decidir o que é melhor para o seu filho.

A passagem da ponte do conhecimento do profissional à mãe é uma tarefa árdua a ser vencida. O profissional deve estar sempre alerta e preparado para modificações na sua rotina e postura e lembrar que, mesmo errando, é importante procurar acertar. Mudar o paradigma do atendimento com a ajuda do aconselhamento em amamentação é um desafio que deve ser enfrentado e vencido.

\section{Referências}

1. Wikipedia the free Encyclopedia, Family (biology) Linnaean taxonomy [homepage on the Internet]. Available at: http:// en.wikipedia.org/wiki/Carolus. Accessed: July 30, 2004.

2. Sokol EJ. Preâmbulo. In: Sokol EJ, editor. Em defesa da amamentação. São Paulo: IBFAN Brasil; 1999. p. 11-8. 
3. Anderson JW, Johnstone BM, Remely DT. Breastfeeding and cognitive development: a meta-analysis. Am J Clin Nutr. $1999 ; 70: 525-35$.

4. Halpern R, Giugliani ERJ, Victora CG, Barros FC, Horta BL. Fatores de risco para suspeita de atraso no desenvolvimento neuropsicomotor aos 12 meses de vida. J Pediatr (Rio J). 2000;76:421-8.

5. Bachrach VR, Schwarz E, Bachrach LR. Breastfeeding and the risk of hospitalization for respiratory disease in infancy: a metaanalysis. Arch Pediatr Adolesc Med. 2003;157:237-43.

6. Duffy LC, Faden H, Wasiewski R, Wolf J, Krystofik D. Exclusive breastfeeding protects against bacterial colonization and day care exposure to otites media. Pediatrics. 1997;100:E7.

7. Jones J, Riley M, Dwyer T. Breastfeeding in early life and bone mass in prepubertal children: a longitudinal study. Osteoporos Int. $2000 ; 11: 146-52$.

8. Lawrence RM. Host-resistance factors and immunologic significance of human milk. In: Lawrence RA, Lawrence RM. Breastfeeding. A guide for the medical profession. 5th ed. St. Louis (MO): CV Mosby; 1999. p. 159-98.

9. Murahovschi J, Teruya KM, Santos Bueno LG, Baldin PE. Profissionais de saúde e a amamentação. In: Amamentação: da teoria à prática. Santos: Fundação Lusíada; 1996. p. 7-40.

10. Ravelli AC, van der Meulen JH, Osmond C, Barker OP. Infant feeding and adult glucose tolerance, lipid profile, blood pressure, and obesity. Arch. Dis Child. 2000;82:248-52.

11. Gdalevich M, Mimouni D, David M, Mimouni M. Breastfeeding and the onset of atopic dermatitis in childhood: a systematic review and meta-analysis of prospective studies. J Am Acad Dermatol. 2001;45:520-7.

12. Gdalevich M, Mimouni D, Mimouni M. Breastfeeding and the risk of bronchial asthma in childhood: a systematic review with meta-analysis of prospective studies. J Pediatr. 2001;139:261-6.

13. Victora CG, Smith PG, Vaughan JP. Evidence for the protection by breast-feeding against infant death from infections diseases in Brazil. Lancet. 1987;2:319-22.

14. World Health Organization Collaborative Study Team on the Role of Breastfeeding on the Prevention of Infant Mortality. How much does breastfeeding protect against infant and child mortality due to infection disease? A pooled analysis of six studies from less developed countries. Lancet. 2000;355:451-5.

15. Bernier MO, Plu-Bureau G, Bossard N, Ayzac L, Thalabard JC. Breastfeeding and risk of breast cancer: a metaanalysis of published studies. Hum Reprod. 2000;6:374-86.

16. World Health Organization, Technical Consultation on Infant and Young Child Feeding. Geneve: World Health Organization; 2002.

17. Ministério da Saúde, Secretaria de Políticas de Saúde, Área de Saúde da Criança. Prevalência de aleitamento materno nas capitais brasileiras e no Distrito Federal. Brasília: Ministério da Saúde; 2001.

18. World Health Organization/UNICEF. Protecting, promoting and supporting breastfeeding: the special role of maternity services. Joint WHO/UNICEF Statement. Geneva; 1989.

19. American Academy of Pediatrics. Breastfeeding and the use of human milk. American Academy of Pediatrics. Work Group on Breastfeeding. Pediatrics. 1997;100:1035-9.

20. American College of Obstetricians and Gynecologists. Breastfeeding: maternal and infant aspects: ACOG Educational Bulletin. Int J Gynaecol Obstet. 2001;74:217-32.
21. Ministério da Saúde. Informe Saúde. Ano 4. no 69. Brasília: Ministério da Saúde; 2000.

22. Réa MF. O pediatra e a amamentação exclusiva. J Pediatr (Rio J). 2003;79:479-80.

23. Alvarado MR, Atalah SE, Diaz FS, Rivero VS, Labbe DM, Escudero PY. Evaluation of a breastfeeding-support programme with health promoters' participation. Food Nutr Bull. 1996;17:49-53.

24. Davies-Adetegbu AA, Adebawa HA. The Ife South Breastfeeding Project: training community health extension workers to promote and manage breastfeeding in rural communities. Bull World Health Organ. 1997;75:323-32.

25. Haider R, Kabir I, Hamadani JD, Habte D. Reasons for failure of breast-feeding counselling: mothers' perspectives in Bangladesh. Bull World Health Organ. 1997;75:191-6.

26. Haider R, Ashworth A, Kabir I, Huttly SR. Effect of communitybased peer counsellors on exclusive breastfeeding practices in Dhaka, Bangladesh: a randomised controlled trial Lancet. 2000;356:1643-7.

27. Lutter CK, Perez-Escamilla R, Segall A, Sanghvi T, Teruya K, Wickham $C$. The effectiveness of a hospital-based program to promote exclusive breast-feeding among low-income women in Brazil. Am J Publ Health. 1997;87:659-63.

28. World Health Organization/UNICEF. Breastfeeding counselling: A training course. Geneva: World Health Organization/UNICEF; 1993.

29. Wang EEL. Breastfeeding. In: Canadian Task Force on the Periodic Health Examination, editor. Canadian guide to clinical preventive health care. Ottawa: Health Canada; 1994. p. 232-42.

30. Guise JM, Palda V, Westhoff C, Chan B, Helfand M, Lieu TA. The effectiveness of primary care-based interventions to promote breastfeeding: systematic evidence review and meta-analysis for the U.S. Preventive Services Task Force. Ann Fam Med. 2003; $1: 70-8$.

31. Palda VA, Guise JM, Wathen CN, Canadian Task Force on Preventive Health Care. Interventions to promote breastfeeding: updated recommendations from the Canadian Task Force on Preventive Health Care. CTFPHC Tech Rep 03-6. London (ON): Canadian Task Force on Preventive Health Care; 2003.

32. Mckinney JP, Fitzgerald HE, Strommen EA. Psicología del desarrollo: edad adolescente. México, D. F.: Ed. Manual Moderno; 1982.

33. Scheeffer R. Aconselhamento psicológico: teoria e prática. São Paulo: Ed. Atlas S.A.; 1989.

34. Rea MF, Venâncio SI. Avaliação do curso de Aconselhamento em Amamentação OMS/UNICEF. J Pediatr (Rio J). 1999;75:112-8.

35. World Health Organization. Evidence for the ten steps to successful breastfeeding. Geneva: World Health Organization/ UNICEF; 1998.

Correspondência:

Keiko Miyasaki Teruya

Rua Piauí, 32

CEP 11065-420 - Santos, SP

Fone: (13) 3202.1355/3237.3322

E-mail: teruyas@uol.com.br 